\title{
5-HTTLPR and BDNF Val66Met polymorphisms and response to rTMS treatment in drug resistant depression
}

\author{
Luisella Bocchio-Chiavetto $^{\mathrm{a}, *}$, Carlo Miniussi ${ }^{\mathrm{a}, \mathrm{b}}$, Roberta Zanardini ${ }^{\mathrm{a}}$, Anna Gazzoli ${ }^{\mathrm{a}}$, \\ Stefano Bignotti ${ }^{a}$, Claudia Specchia ${ }^{c}$, Massimo Gennarelli ${ }^{\mathrm{a}, \mathrm{d}}$ \\ a I.R.C.C.S. “San Giovanni di Dio", Fatebenefratelli, Via Piastroni 4, Brescia, Italy \\ b Physiology, Department of Biomedical Sciences and Biotechnologies, University of Brescia, Viale Europa 11, Brescia, Italy \\ ' Medical Statistics, Department of Biomedical Sciences and Biotechnologies, University of Brescia, Viale Europa 11, Brescia, Italy \\ d Biology and Genetics, Department of Biomedical Sciences and Biotechnologies, University of Brescia, Viale Europa 11, Brescia, Italy
}

\section{A R T I C L E I N F O}

\section{Article history:}

Received 29 January 2008

Received in revised form 28 March 2008

Accepted 2 April 2008

\section{Keywords:}

5-HTTLPR

BDNF

Depression

rTMS

Val66Met

Antidepressant

\begin{abstract}
A B S T R A C T
Repetitive transcranial magnetic stimulation (rTMS) is a painless and safe brain stimulation technique that has been found to be effective in treating depression symptoms. The potential usefulness of rTMS, in particular to treat drug resistant patients, might be increased by identifying genetic predictors of efficacy. According to this rationale, we investigated the role of two functional polymorphisms in the genes coding for the serotonin transporter (5-HTTLPR) and the brain-derived neurotrophic factor (BDNF Val66Met), and rTMS response in a group of 36 drug resistant patients affected by mood disorders. rTMS treatment significantly improved depression symptomatology $(p<0.0001)$ and the response was significantly greater in 5-HTTLPR LL homozygotes compared to S allele carriers $(p=0.007)$ and in BDNF Val/Val homozygotes compared to Met allele carriers $(p=0.024)$. These findings provide evidences about the involvement of both polymorphisms in rTMS antidepressant response. Further investigations in larger samples are needed to clarify the usefulness of 5-HTTLPR and BDNF Val66Met genotyping in the optimization of non-pharmacological treatments in mood disorders.
\end{abstract}

(c) 2008 Elsevier Ireland Ltd. All rights reserved.
Transcranial magnetic stimulation (TMS) consists of a brief and intense magnetic field, created by a strong electric current circulating within a coil resting on the scalp, that penetrates human tissues painlessly and safely inducing electric currents that can depolarize neurons or their axons. As a result, this may induce a subsequent activation of the connected cortico-cortical and cortico-subcortical networks. In its repetitive form (rTMS), a train of electromagnetic pulses is applied on the scalp provoking, if repeated over several days, a relatively long-lasting change in excitability. During the last decade, rTMS showed evidences of antidepressant effectiveness as an add-on therapy to treat drug resistant mood disorders $[5,8,16,26]$, although reviews and meta-analyses reported low rates of clinical response and several methodological limitations [9,21]. The biological correlates of the antidepressant effectiveness have not been completely clarified, even though several evidences have suggested common mechanisms of action with antidepressant drugs and electroconvulsive therapies. The

\footnotetext{
* Corresponding author at: Neuropsychopharmacology Unit, I.R.C.C.S. “San Giovanni di Dio", Fatebenefratelli, Via Pilastroni 4, 25123 Brescia, Italy. Tel.: +39030 3501453; fax: +390303533513.

E-mail address: lbocchio@fatebenefratelli.it (L. Bocchio-Chiavetto).
}

SLC6A4 gene contains a functional polymorphism (5-HTTLPR, 44bp insertion/deletion) resulting in a long $(\mathrm{L}) / \operatorname{short}(\mathrm{S})$ variant in the promoter region upstream of the transcription starting site. The $\mathrm{S}$ allele determines decreased transcriptional activity and has been associated with an increased susceptibility to develop mood disorders in response to stressful life events [6], as well as with poorer outcomes after antidepressant pharmacological and nonpharmacological treatments $[3,30]$. In addition, a recent study evidenced a significant influence of the 5-HTTLPR polymorphism on treatment outcome both after active and sham rTMS stimulations [35].

A functional polymorphism (SNP rs6265), producing a valine (Val) to methionine (Met) substitution at the codon 66 (Val66Met) in the proBDNF region, has been characterized in the BDNF gene, exhibiting a detrimental effect on intracellular trafficking and activity-dependent secretion and influencing hippocampal function, episodic memory and brain morphology [11,2]. Despite a critical mass of evidences in clinical and animal model studies that support a role of this neurotrophin in mood disorders [22], recent meta-analyses do not indicate an involvement of the BDNF Val66Met polymorphism in the susceptibility [15]. More probably, the polymorphism might play a role in subgroups of patients, homogeneous for cluster of symptoms and clinical course [28], 
exposure to other genetic or environmental risk factors [19], or resistance to drug treatment [1].

In this context, in order to investigate the potential of a "pharmacogenetic approach" when tailoring non-pharmacological treatments for mood disorders, we have analyzed possible associations between the two functional polymorphisms in the SLC6A4 and BDNF genes and the response to rTMS in a group of drug resistant patients affected by mood disorders.

Thirty-six patients (29 females, 7 males; mean age \pm S.D.: $59.67 \pm 13.24$ years; mean age at onset \pm S.D.: $40.35 \pm 15.03$ years) with depression symptomatology/symptoms planning to receive rTMS treatment were enrolled in the study by the Psychiatric Unit of IRCCS Fatebenefratelli (Brescia, Italy), after the approval of the study protocol by the local Ethics Committee and the obtainment of their informed consents. All subjects were of Caucasoid ancestry and were living in the Northern Italy. The DSM IV diagnoses were major depression for 31 subjects and bipolar disorder (depressive phase) for five subjects and were formulated, by an expert psychiatrist after meticulous diagnostic interviews. Severity of illness was assessed using the 21-item Hamilton rating scale for depression (HAMD). Criteria for inclusion were as follow: baseline HAMD $\left(\mathrm{HAMD}_{0}\right) \geq 17$ and resistance to drug treatment defined as failure to response in the current episode to at least two classes of antidepressants (courses of at least 8 weeks at standard dosages) (stage II definition: [32]). Exclusion criteria were pregnancy, major medical or neurological disorders beyond the exclusion criteria for rTMS [33].

The patients were deliberately not withdrawn from their current drug treatment before the trial, and they were kept under constant medication for 3 months prior to the enrolment and for the entire study duration. In particular, 24 patients were receiving mono- or combined therapies with selective serotonin reuptake inhibitors (SSRIs), while 12 subjects were taking other antidepressants. The mean dose as imipramine equivalents was $148.56 \pm 49.77$. Twelve patients were receiving a concomitant treatment with typical (seven subjects) and atypical antipsychotics (five subjects).

All the 36 subjects underwent an active rTMS treatment as described elsewhere [23]. In particular, frequency of stimulation was $1 \mathrm{~Hz}$ for 18 subjects and $17 \mathrm{~Hz}$ for the other 18 subjects; these two frequencies had been previously found to have similar effectiveness on depression [23]. rTMS treatment consisted of five consecutive sessions of stimulation (in the morning) separated by $24 \mathrm{~h}$.

A subgroup of 15 subjects (not different in any clinical or demographic variable from the subgroup of 21 subjects that received only active rTMS) underwent also a sham treatment as they have been included in a clinical trial setting with a double-blind, placebocontrolled, cross-over design [23]. The two blocks of active and sham stimulations were separated by an interval of 8 weeks: six patients received real stimulation first, and nine first underwent ineffective sham stimulation. In the sham treatment, a $25 \mathrm{~mm}$ thick plywood shield, build to appear as an integral part of the apparatus, was interposed between the coil itself and the scalp, separating the two ones. The ventral surface of the coil was upside down and stimulus intensity was substantially decreased at $60 \%$ below motor threshold. This 'placebo condition' has been shown completely ineffective in inducing cortical excitability [23].

Before starting the first stimulation session $\left(\mathrm{HAMD}_{0}\right)$, and after the last one $\left(\mathrm{HAMD}_{1}\right)$, clinical evaluations were performed and severity of illness was assessed by HAMD scores. Response to treatment was defined as percentage decrease in HAMD scores (\%HAMD). An expert psychiatrist blind to the treatment performed the ratings.

A blood sample for DNA extraction was taken from each subject in the morning of the first day of treatment.
Genomic DNA was isolated using a commercially available kit (GENTRA).

At the end of all clinical evaluations, genotyping of 5-HTTLPR and BDNF Val66Met was conducted as previously described [17,24]. Demographic and clinical characteristics in the samples stratified for genotype were described either in terms of mean \pm S.D. if quantitative, or in proportional terms. After checking for normality, paired and Student's $t$-tests were used when appropriate to evaluate differences in quantitative variables. Qualitative variables were tested by means of $\chi^{2}$ and Fisher tests.

\%HAMD variation in treatment response was evaluated in a linear regression model. Every demographic, clinical and genetic covariate was tested in a univariate analysis. A multiple regression was used to examine the simultaneous contribution of the significant covariates and their interaction to the outcome variable. Statistical analyses were performed using SPSS, Version 13.0 (website: http://www.spss.com). Power analysis was conducted using the software QUANTO Version 1.2 (website: http://hydra.usc.edu/gxe).

Active rTMS treatment significantly improved depression symptomatology in the whole study group of 36 subjects (mean values \pm S.D.: $\mathrm{HAMD}_{0}=23.19 \pm 5.12 ; \mathrm{HAMD}_{1}=17.50 \pm 6.91 ; t=6.51$, $p<0.0001$ ) with an average percentage improvement of $25.29 \%$ (\%HAMD). No demographic (sex, age) or clinical variables (diagnosis, age at onset, number of previous treatments, $\mathrm{HAMD}_{0}$ scores, type of drug SSRIs/others, dosage as imipramine equivalents, antipsychotic treatment, and rTMS protocol $1 \mathrm{~Hz} / 17 \mathrm{~Hz}$ ) were found to be statistically associated with rTMS response at univariate analysis.

Genotype distributions were: $\mathrm{LL}=10, \mathrm{SL}=18$, and $\mathrm{SS}=8$ for 5 -HTTLPR polymorphism; and $\mathrm{Val} / \mathrm{Val}=20, \mathrm{Met} / \mathrm{Val}=14$, and Met/Met $=2$ for BDNF Val66Met. Genotypic groups were compared considering a dominant effect of minor alleles for both polymorphisms, hypothesized on the basis of evidences coming from functional studies on cortical excitability and plasticity $[12,20]$ and data from studies on antidepressant response [30]. As shown in Table 1, no significant differences were observed in demographic characteristics (age, sex), clinical features (diagnosis, age at onset, number of previous treatments, $\mathrm{HAMD}_{0}$ scores), types of pharmacological treatment during the study (SSRIs vs. other antidepressants, dosage as imipramine equivalents, concomitant antipsychotic treatments), and rTMS treatment protocols $(1 \mathrm{~Hz}$ vs. $17 \mathrm{~Hz})$ in the samples defined by 5-HTTLPR and BDNF Val66Met genotypes (major allele homozygotes vs. minor allele carriers). (Fig. 1a: 5-HTTLPR LL homozygote 10 subjects: \%HAMD $=40.49 \pm 25.57$; S carrier 26 subjects: \%HAMD $=19.44 \pm 17.51$; Fig. $1 \mathrm{~b}$ : BDNF Val/Val homozygote 20 subjects: \%HAMD $=32.36 \pm 21.23$; Met carrier 16 subjects: $\% \mathrm{HAMD}=16.45 \pm 19.90$.) Univariate regression analysis resulted in a significant increase in percentage symptom improvement after rTMS treatment for both 5-HTTLPR LL and BDNF Val/Val homozygotes (5-HTTLPR S carriers: $r^{2}=0.191, \beta=-0.437, p=0.008$; BDNF Met carriers: $r^{2}=0.134, \beta=-0.366, p=0.028$ ). Multiple linear regression, simultaneously taking into account both 5-HTTLPR $\mathrm{S}$ carrier and BDNF Met carrier genotypes, showed an independent and more significant effect of both polymorphisms on \%HAMD reduction (5-HTTLPR S carriers: $r^{2}=0.308, \beta=-0.418, p=0.007$; BDNF Met carriers: $r^{2}=0.308, \beta=-0.343, p=0.024$ ) after rTMS treatment. Finally, the interaction between genotypes carrying minor alleles of both polymorphisms was tested in the regression model and was found to be not significant.

The independent effects of both polymorphisms were confirmed also in a extended analysis of multiple linear regression considering the effects of main covariates (sex, diagnosis, rTMS protocol, imipramine equivalents, antipsychotic treatment) (see Table A Supplementary Results). 
Table 1

Patient demographic and clinical characteristics in the sample stratified for 5-HTTLPR and BDNF Val66Met genotypes

\begin{tabular}{|c|c|c|c|c|c|c|}
\hline & \multicolumn{3}{|l|}{ 5-HTTLPR } & \multicolumn{3}{|l|}{ BDNF Val66Met } \\
\hline & LL & S carriers & $p$ & Val/Val & Met carriers & $p$ \\
\hline Number of subjects & 10 & 26 & & 20 & 16 & \\
\hline \multicolumn{7}{|l|}{ Diagnosis } \\
\hline Major depression & 8 & 23 & & 18 & 13 & \\
\hline Bipolar disorder & 2 & 3 & & 2 & 3 & \\
\hline Age $^{a}$ & $58.20 \pm 14.98$ & $60.23 \pm 12.79$ & 0.686 & $56.10 \pm 13.71$ & $64.13 \pm 11.53$ & 0.070 \\
\hline Sex $(\text { males })^{\mathrm{b}}$ & $3(0.30)$ & $4(0.15)$ & 0.370 & $3(0.15)$ & $4(0.25)$ & 0.675 \\
\hline Age at onset ${ }^{\mathrm{a}}$ & $41.70 \pm 12.82$ & $39.79 \pm 16.08$ & 0.741 & $37.30 \pm 14.80$ & $44.71 \pm 14.76$ & 0.160 \\
\hline Previous treatments ${ }^{\mathrm{a}}$ & $2.80 \pm 0.79$ & $2.92 \pm 1.21$ & 0.782 & $2.79 \pm 0.98$ & $3.00 \pm 1.25$ & 0.585 \\
\hline $\mathrm{HAMD}_{0}{ }^{\mathrm{a}}$ & $23.40 \pm 6.64$ & $23.12 \pm 4.56$ & 0.884 & $24.10 \pm 5.60$ & $22.06 \pm 4.34$ & 0.241 \\
\hline Antidepressants (SSRIs/others) ${ }^{\mathrm{b}}$ & $6(0.60)$ & $18(0.69)$ & 0.599 & $14(0.70)$ & $10(0.63)$ & 0.635 \\
\hline Imipramine equivalents ${ }^{a}$ & $155.79 \pm 55.97$ & $145.78 \pm 48.08$ & 0.596 & $137.03 \pm 41.73$ & $162.95 \pm 56.35$ & 0.122 \\
\hline Antipsychotics $^{\mathrm{b}}$ & $3(0.30)$ & $9(0.34)$ & 1.00 & $6(0.30)$ & $6(0.37)$ & 0.635 \\
\hline rTMS protocol $(17 \mathrm{~Hz}: 1 \mathrm{~Hz})^{\mathrm{b}}$ & $(5: 5)$ & $(13: 13)$ & 1.00 & $(10: 10)$ & $(8: 8)$ & 0.643 \\
\hline
\end{tabular}

a Mean value \pm S.D.

b Frequency.

Given our sample size, a post hoc analysis based on the allele frequencies calculated from the sample genotype distribution revealed a power of $81.33 \%$ and $65.39 \%$, respectively to evidence the observed effects on \%HAMD reduction for 5-HTTLPR and BDNF Val66Met minor allele carriers.

The subgroup of subjects that underwent also the sham rTMS treatment showed an average symptom improvement of $13.05 \%$ ( mean values \pm S.D.: $\mathrm{HAMD}_{0}=24.53 \pm 4.79 ; \mathrm{HAMD}_{1}=21.13 \pm 4.53$; $t=3.37, p<0.01$ ). No differences in \%HDRS score have been found after sham treatment, between carrier and non-carrier of minor alleles for either polymorphisms (5-HTTLPR LL homozygote three subjects: \%HAMD $=8.78 \pm 4.23, \mathrm{~S}$ carrier 12 subjects: $\% \mathrm{HAMD}=14.11 \pm 16.83, F=0.281, p=0.605$; BDNF Val/Val homozygote 10 subjects: $\% \mathrm{HAMD}=16.52 \pm 10.64$, Met carrier five subjects: $\%$ HAMD $=6.11 \pm 21.46, F=1.640, p=0.223$ ).

The present findings provide direct evidence of a role of the 5-HTTLPR and BDNF Val66Met functional polymorphisms in the improvement of depression symptoms after rTMS treatment. In particular, the response to rTMS was increased (average improvement > 30\%) in 5-HTTLPR LL and BDNF Val/Val homozygote subjects for the genetic variants associated with increased protein activity. The effects of the two polymorphisms in our sample were inde- pendent and no other demographic and clinical variable was found to affect rTMS response. On the contrary, no influence of either polymorphism on symptom improvement was observed after sham treatment in a subsample of the same subjects.

The findings on the influence of BDNF Val66Met on rTMS treatment were novel evidences, while the data on 5-HTTLPR were partially overlapping recent results [35], obtained in sample of 99 subject that merged together drug resistant and non-resistant patients from two randomized studies on rTMS effectiveness as add-on therapy to pharmacological treatment. In this study the authors reported an involvement of the polymorphism in the response to treatment after both active and sham rTMS stimulations. The divergences in the influence of 5-HTTLPR on the response to sham treatment could be explained considering the different experimental settings: the cross-over design of our study on drug resistant patients in stable pharmacological treatment for at least 3 months before the enrolment might be more adequate to avoid spurious effects of the polymorphism on the response to concomitant drug treatment and on depressive episode length in subjects receiving the placebo treatment, as suggested by [35] and colleagues. However, our data on sham response are only explorative considering the reduced group of subject that underwent also the inactive
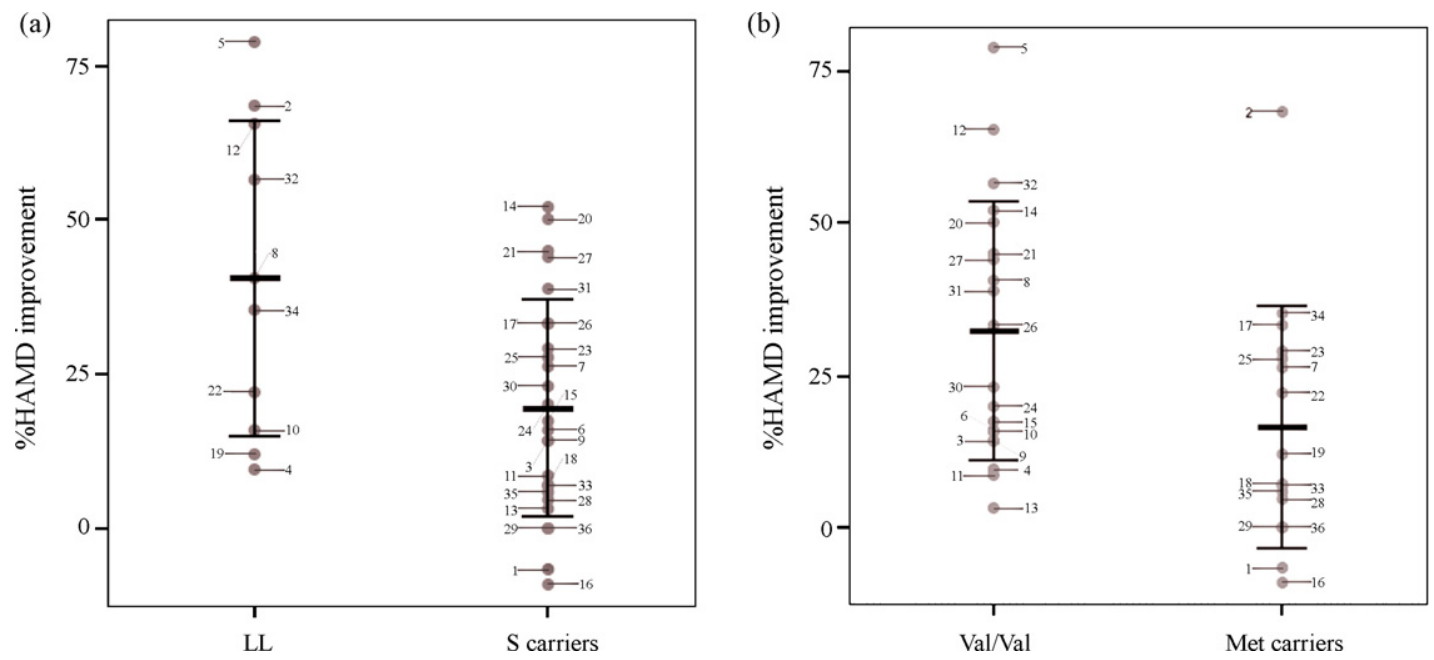

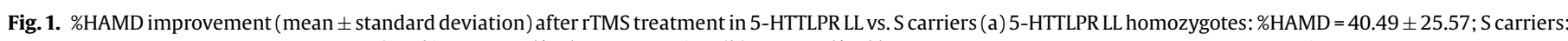

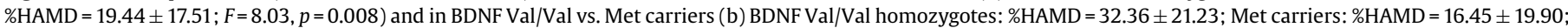
$F=5.27, p=0.028$ ). Label $=$ subject code. 
treatment and not allow us to exclude an effect of both polymorphisms on the placebo response.

These genetic variants might influence the response to treatment by affecting molecular rTMS targets. In animal models, rTMS treatment was found to be able to regulate serotonin neurotransmission through the modulation of SLC6A4 mRNA expression [18], a process that might be facilitated by the presence of the 5HTTLPR L allele. In parallel, both clinical [36] and preclinical studies [25] evidenced a stimulatory effect of rTMS treatment on BDNF expression and synthesis that might be enhanced by the presence of the Val variant associated with better functionality [11] Together with their actions on the dynamic profiles, 5-HTTLPR and BDNF Val66Met polymorphisms might contribute to individual differences in rTMS treatment outcome also influencing brain morphological and functional variability $[2,29]$. 5-HTTLPR S carriers showed decreased anterior cingulate cortex and amygdale volumes, increased volumes in the pulvinar nucleus of the thalamus, as well as functional alterations in limbic circuits, striatal and cortical regions [4,34]. Furthermore, the 5-HTTLPR genotype influenced neural response in the anterior cingulate and dorsolateral prefrontal cortex in depressed patients treated with sleep deprivation combined with light therapy [3]. Similarly, the BDNF Met allele was associated with volume decreases in cortical regions in healthy subjects [27] and in transgenic mice [7]. The prefrontal cortex is involved in mood regulation and, generally, unbalanced functioning of the two cortices has been associated with depression symptoms [10], while the ability to non-invasively excite or inhibit focal cortical areas with TMS has been demonstrated by several studies [31].

Based upon the above, we hypothesize that 5-HTTLPR and BDNF Val66Met polymorphisms might influence rTMS effectiveness by inducing modifications in brain cortical excitability at the site of stimulation as well as in related areas. In this regard, rTMS measures performed in functional studies evidenced differences in cortical experience-dependent plasticity in subjects stratified for BDNF Val66Met polymorphism [20], while 5-HTTLPR LL healthy volunteers treated with citalopram showed increased motor cortex excitability [12]. However, these results should be considered as explorative, since some limitations to the study should be taken into account. In particular, the study sample size was limited. Nevertheless, a post hoc analysis estimated the sample power to be adequate at least for the main analysis on 5-HTTLPR polymorphism, given the magnitude of the observed effect on \%HAMD reduction for 5-HTTLPR minor allele carriers. Moreover, the group of patients was heterogeneous, comprising unipolar and bipolar depressed patients with a complex symptomatology, in treatment with different antidepressants. However, the preliminary analysis on our sample revealed that no clinical or demographic characteristics were statistically associated with rTMS response or were differentially distributed among genotypes. Finally, rTMS seems to be effective in treating depression [14], though [13] suggest that at least 4 weeks of treatment are necessary to achieve clinically meaningful benefit. Our experimental protocol was indeed brief (only 5 days) compare to these suggestions, nevertheless we provided that a 5-day treatment was clinically more effective in patients carrying the 5-HTTLPR LL and BDNF Val/Val genotypes, and this may be an important factor to decide treatment duration in future protocols.

In conclusion, further investigations in larger samples with longer follow-up are needed to go beyond these limitations and to evaluate the usefulness in the clinical practice of 5-HTTLPR and BDNF Val66Met genotyping to predict rTMS response. Replications in larger samples will also permit to extend genetic analyses to other polymorphisms in the same genes and to evaluate putative gene-gene synergic effects and interactions with other clinical predictors of effectiveness. Our results provide new findings for the comprehension of biological mechanisms underlying rTMS treatment of depression symptoms and suggest the potential usefulness of "pharmacogenetic" investigation strategies in the optimization of non-pharmacological treatments in mood disorders.

\section{Acknowledgement}

This research was supported by a grant from the Italian Ministry of Health.

\section{Appendix A. Supplementary data}

Supplementary data associated with this article can be found, in the online version, at doi:10.1016/j.neulet.2008.04.005.

\section{References}

[1] S. Anttila, K. Huuhka, M. Huuhka, R. Rontu, M. Hurme, E. Leinonen, T. Lehtimaki, Interaction between 5-HT1A and BDNF genotypes increases the risk of treatment-resistant depression, J. Neural. Transm. 114 (2007) 1065-1068.

[2] K.G. Bath, F.S. Lee, Variant BDNF (Val66Met) impact on brain structure and function, Cogn. Affect. Behav. Neurosci. 6 (2006) 79-85.

[3] F. Benedetti, A. Bernasconi, V. Blasi, M. Cadioli, C. Colombo, A. Falini, C. Lorenzi, D. Radaelli, G. Scotti, E. Smeraldi, Neural and genetic correlates of antidepressant response to sleep deprivation: a functional magnetic resonance imaging study of moral valence decision in bipolar depression, Arch. Gen. Psychiatry 64 (2007) 179-187.

[4] T. Canli, K. Omura, B.W. Haas, A. Fallgatter, R.T. Constable, K.P. Lesch, Beyond affect: a role for genetic variation of the serotonin transporter in neural activation during a cognitive attention task, Proc. Natl. Acad. Sci. U.S.A. 102 (2005) $12224-12229$.

[5] L.L. Carpenter, Neurostimulation in resistant depression, J. Psychopharmacol. 20 (2006) 35-40.

[6] A. Caspi, K. Sugden, T.E. Moffitt, A. Taylor, I.W. Craig, H. Harrington, J. McClay, J. Mill, J. Martin, A. Braithwaite, R. Poulton, Influence of life stress on depression: moderation by a polymorphism in the 5-HTT gene, Science 301 (2003) 386389.

[7] Z.Y. Chen, D. Jing, K.G. Bath, A. Ieraci, T. Khan, C.J. Siao, D.G. Herrera, M. Toth, C. Yang, B.S. McEwen, B.L. Hempstead, F.S. Lee, Genetic variant BDNF (Val66Met) polymorphism alters anxiety-related behavior, Science 14 (2006) 140-143.

[8] CME Institute of Physicians Postgraduate Press Inc., Transcranial magnetic stimulation: potential new treatment for resistant depression, J. Clin. Psychiatry 68 (2007) 315-330.

[9] J.L. Couturier, Efficacy of rapid-rate repetitive transcranial magnetic stimulation in the treatment of depression: a systematic review and meta-analysis, J. Psychiatry Neurosci. 30 (2005) 83-90

[10] R.J. Davidson, What does the prefrontal cortex "do" in affect: perspectives on frontal EEG asymmetry research, Biol. Psychol. 67 (2004) 219-233.

[11] M.F. Egan, M. Kojima, J.H. Callicott, T.E. Goldberg, B.S. Kolachana, A. Bertolino, E. Zaitsev, B. Gold, D. Goldman, M. Dean, B. Lu, D.R. Weinberger, The BDNF val66met polymorphism affects activity-dependent secretion of BDNF and human memory and hippocampal function, Cell 112 (2003) 257-269.

[12] P. Eichhammer, B. Langguth, R. Wiegand, A. Kharraz, U. Frick, G. Hajak, Allelic variation in the serotonin transporter promoter affects neuromodulatory effects of a selective serotonin transporter reuptake inhibitor (SSRI), Psychopharmacology 166 (2003) 294-297.

[13] P.B. Fitzgerald, T.L. Brown, N.A. Marston, Z.J. Daskalakis, A. De Castella, J. Kulkarni, Transcranial magnetic stimulation in the treatment of depression: a double-blind, placebo-controlled trial, Arch. Gen. Psychiatry 60 (2003) 1002-1008.

[14] A.A. Gershon, P.N. Dannon, L. Grunhaus, Transcranial magnetic stimulation in the treatment of depression, Am. J. Psychiatry 160 (2003) 835-845.

[15] M. Gratacos, J.R. Gonzalez, J.M. Mercader, R. de Cid, M. Urretavizcaya, X. Estivill, Brain-derived neurotrophic factor Val66Met and psychiatric disorders: meta-analysis of case-control studies confirm association to substance-related disorders, eating disorders, and schizophrenia, Biol. Psychiatry 61 (2007) 911-922.

[16] M. Gross, L. Nakamura, A. Pascual-Leone, F. Fregni, Has repetitive transcranial magnetic stimulation (rTMS) treatment for depression improved? A systematic review and meta-analysis comparing the recent vs. the earlier rTMS studies, Acta Psychiatr. Scand. 116 (2007) 165-173.

[17] A. Heils, A. Teufel, S. Petri, G. Stober, P. Riederer, D. Bengel, K.P. Lesch, Allelic variation of human serotonin transporter gene expression, J. Neurochem. 66 (1996) 2621-2624.

[18] T. Ikeda, M. Kurosawa, C. Uchikawa, S. Kitayama, N. Nukina, Modulation of monoamine transporter expression and function by repetitive transcranial magnetic stimulation, Biochem. Biophys. Res. Commun. 327 (2005) 218-224.

[19] J. Kaufman, B.Z. Yang, H. Douglas-Palumberi, D. Grasso, D. Lipschitz, S. Houshyar, J.H. Krystal, J. Gelernter, Brain-derived neurotrophic factor-5-HTTLPR gene 
interactions and environmental modifiers of depression in children, Biol. Psychiatry 59 (2006) 673-680.

[20] J.A. Kleim, S. Chan, E. Pringle, K. Schallert, V. Procaccio, R. Jimenez, S.C. Cramer, BDNF val66met polymorphism is associated with modified experiencedependent plasticity in human motor cortex, Nat. Neurosci. 9 (2006) 735-737.

[21] C.K. Loo, P.B. Mitchell, A review of the efficacy of transcranial magnetic stimulation (TMS) treatment for depression, and current and future strategies to optimize efficacy, J. Affect. Disord. 88 (2005) 255-267.

[22] K. Martinowich, B. Lu, Interaction between BDNF and Serotonin: role in mood disorders, Neuropsychopharmacology, doi:10.1038/sj.npp.1301571. Online first September 19, 2007.

[23] C. Miniussi, C. Bonato, S. Bignotti, A. Gazzoli, M. Gennarelli, P. Pasqualetti, G.B. Tura, M. Ventriglia, P.M. Rossini, Repetitive transcranial magnetic stimulation (rTMS) at high and low frequency: an efficacious therapy for major drug-resistant depression? Clin. Neurophysiol. 116 (2005) 1062-1071.

[24] P. Monteleone, R. Zanardini, A. Tortorella, M. Gennarelli, E. Castaldo, B. Canestrelli, M. Maj, The 196G/A (val66met) polymorphism of the BDNF gene is significantly associated with binge eating behaviour in women with bulimia nervosa or binge eating disorder, Neurosci. Lett. 406 (2006) 133-137.

[25] M.B. Muller, N. Toschi, A.E. Kresse, A. Post, M.E. Keck, Long-term repetitive transcranial magnetic stimulation increases the expression of brain-derived neurotrophic factor and cholecystokinin mRNA, but not neuropeptide tyrosine mRNA in specific areas of rat brain, Neuropsychopharmacology 23 (2000) 205-215.

[26] J.P. O'reardon, H.B. Solvason, P.G. Janicak, S. Sampson, K.E. Isenberg, Z. Nahas, W.M. McDonald, D. Avery, P.B. Fitzgerald, C. Loo, M.A. Demitrack, M.S. George, H.A. Sackeim, Efficacy and safety of transcranial magnetic stimulation in the acute treatment of major depression: a multisite randomized controlled trial, Biol. Psychiatry 62 (2007) 1208-1216.

[27] L. Pezawas, B.A. Verchinski, V.S. Mattay, J.H. Callicott, B.S. Kolachana, R.E. Straub, M.F. Egan, A. Meyer-Lindenberg, D.R. Weinberger, The brain-derived neurotrophic factor val66met polymorphism and variation in human cortical morphology, J. Neurosci. 24 (2004) 10099-10102.

[28] R.M. Post, Role of BDNF in bipolar and unipolar disorder: clinical and theoretical implications, J. Psychiatr. Res. 41 (2007) 979-990.

[29] A. Serretti, R. Calati, L. Mandelli, D. De Ronchi, Serotonin transporter gene variants and behavior: a comprehensive review, Curr. Drug Targets 7 (2006) 1659-1669.

[30] A. Serretti, M. Kato, D. De Ronchi, T. Kinoshita, Meta-analysis of serotonin transporter gene promoter polymorphism (5-HTTLPR) association with selective serotonin reuptake inhibitor efficacy in depressed patients, Mol. Psychiatry 12 (2007) 247-257.

[31] H.R. Siebner, J. Rothwell, Transcranial magnetic stimulation: new insights into representational cortical plasticity, Exp. Brain Res. 148 (2003) 1-16.

[32] M.E. Thase, A.J. Rush, When at first you don't succeed: sequential strategies for antidepressant nonresponders, J. Clin. Psychiatry 58 (1997) 23-29.

[33] E.M. Wassermann, Risk and safety of repetitive transcranial magnetic stimulation: report and suggested guidelines from the International Workshop on the Safety of Repetitive Transcranial Magnetic Stimulation, June 5-7, 1996 Electroencephalogr. Clin. Neurophysiol. 108 (1998) 1-16.

[34] K.A. Young, L.A. Holcomb, W.L. Bonkale, B.P. Hicks, U. Yazdani, D.C. German, 5HTTLPR polymorphism and enlargement of the pulvinar: unlocking the backdoor to the limbic system, Biol. Psychiatry 61 (2007) 813-818.

[35] R. Zanardi, L. Magri, D. Rossini, A. Malaguti, S. Giordani, C. Lorenzi, A. Pirovano, E. Smeraldi, A. Lucca, Role of serotonergic gene polymorphisms on response to transcranial magnetic stimulation in depression, Eur. Neuropsychopharmacol. 17 (2007) 651-657.

[36] R. Zanardini, A. Gazzoli, M. Ventriglia, J. Perez, S. Bignotti, P.M. Rossini, M. Gennarelli, L. Bocchio-Chiavetto, Effect of repetitive transcranial magnetic stimulation on serum brain derived neurotrophic factor in drug resistant depressed patients, J. Affect. Disord. 91 (2006) 83-86. 